\title{
Rationale and Architecture for Incorporating Human Oculomotor Plant Features in User Interest Modeling
}

\author{
Sampath Jayarathna and Frank Shipman \\ Computer Science \& Engineering, Texas A\&M University \\ College Station, TX 77843-3112 \\ (Sampath, shipman)@cse.tamu.edu
}

\begin{abstract}
We present a conceptual framework expanding the use of eye movement as a source of implicit relevance feedback. While gaze time has been the primary feature to be incorporated in interest modeling, this work constructs a model of human oculomotor plant features during user's interaction with multiple everyday applications with the goal of better interpreting user gaze data. The following presents the anatomical reasoning behind incorporating additional gaze features, the integration of the additional features into an existing interest modeling architecture, and a plan for assessing the impact of the addition of the features.
\end{abstract}

\section{Keywords}

User interest modeling, eye movements, oculomotor plant, implicit feedback

\section{INTRODUCTION}

Accurate models of user interest are valuable in personalizing the presentation of the often large quantity of information relevant to a query or other form of information request. We have previously developed and evaluated a software framework for capturing user activity across multiple applications and combining this activity data in a user interest model to aid information delivery. In this paper, we present the extension of this user modeling framework based on the non-visible anatomical structure and its characteristics of the human eye.

The human eye already provides a plethora of information useful for user modeling. Eye tracking has been employed in studying user attention on the web. Yarbus et al. show that visual behavior is closely correlated to the user task when looking at a visual scene [23]. A variety of uses of gaze data have been explored as a source of implicit relevance feedback for IR. Previous work has primarily focused on identifying visual attention to make user interest predictions $[1,3]$, interactive image retrieval $[5,9,16]$ and more recently to predict a target in search results $[2,8,20,24]$. In cognitive and perceptual psychology, eye tracking has been established as a mean of analyzing user attention as well as information processing tasks [21]. The human-computer interaction and information retrieval communities have shown that eye-tracking

Permission to make digital or hard copies of all or part of this work for personal or classroom use is granted without fee provided that copies are not made or distributed for profit or commercial advantage and that copies bear this notice and the full citation on the first page. Copyrights for components of this work owned by others than ACM must be honored. Abstracting with credit is permitted. To copy otherwise, or republish, to post on servers or to redistribute to lists, requires prior specific permission and/or a fee. Request permissions from Permissions@acm.org.

CHIIR '16, March 13-17, 2016, Carrboro, NC, USA

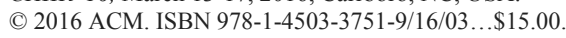

DOI: http://dx.doi.org/10.1145/2854946.2854992 have investigated trends and differences in user attention patterns and cognitive processing including correlations to the relevance of reading text [4], identifying pattern differences [7], tasks types [10], and user accuracy in alternative interfaces [19].

\subsection{Motivation and Hypothesis}

The motivation behind our work is that not all gazes are created equal. Thus, systems should not treat focus activity equally when using gaze information for user interest modeling. We hypothesize that the anatomical characteristics of the human eye may be utilized to estimate oculomotor plant features that can then be used to alter user interest modeling techniques. The research presented here presents a more comprehensive description of oculomotor plant features and an initial hypothesis as to what features will be of most use in conjunction with traditional implicit relevance feedback indicators. To the best of our knowledge, very few studies have used oculomotor plant characteristics $[14,15]$ in the context of biometrics. This study attempts to use eye movements and these oculomotor plant characteristics to identify the relevance of content.

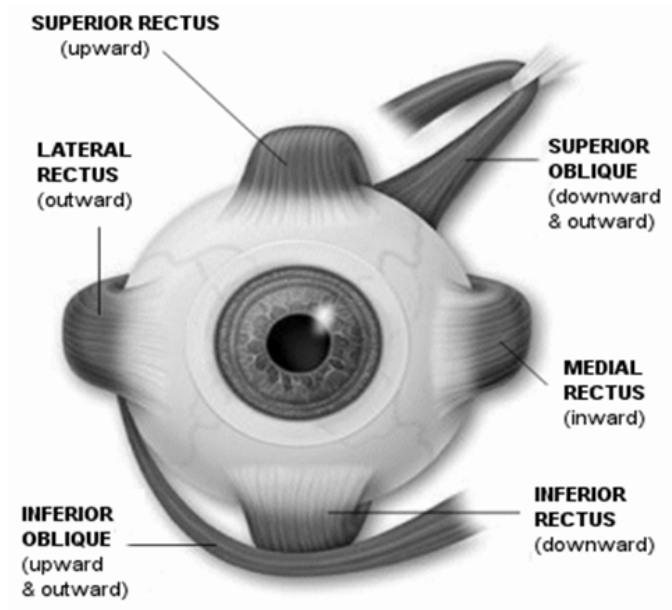

Figure 1. Human Oculomotor Plant

\section{HUMAN OCULOMOTOR PLANT}

The human oculomotor plant (OP) consists of the eye globe and six extraocular muscles and its surrounding tissues, ligaments each containing thick and thin filaments, tendon-like components and liquids (see Figure 1). In general there are six major eye movement types: fixations, saccades, smooth pursuits, optokinetic reflex, vestibule-ocular reflex and vergence [17]. An eye-tracker provides eye gaze position information as well as other gaze related parameters (pupil dilation etc.) so that algorithmic derivation in terms of two primary eye movements, fixations (relative gaze position at one point on the screen), and saccades (rapid eye movements of gaze from one fixation point 

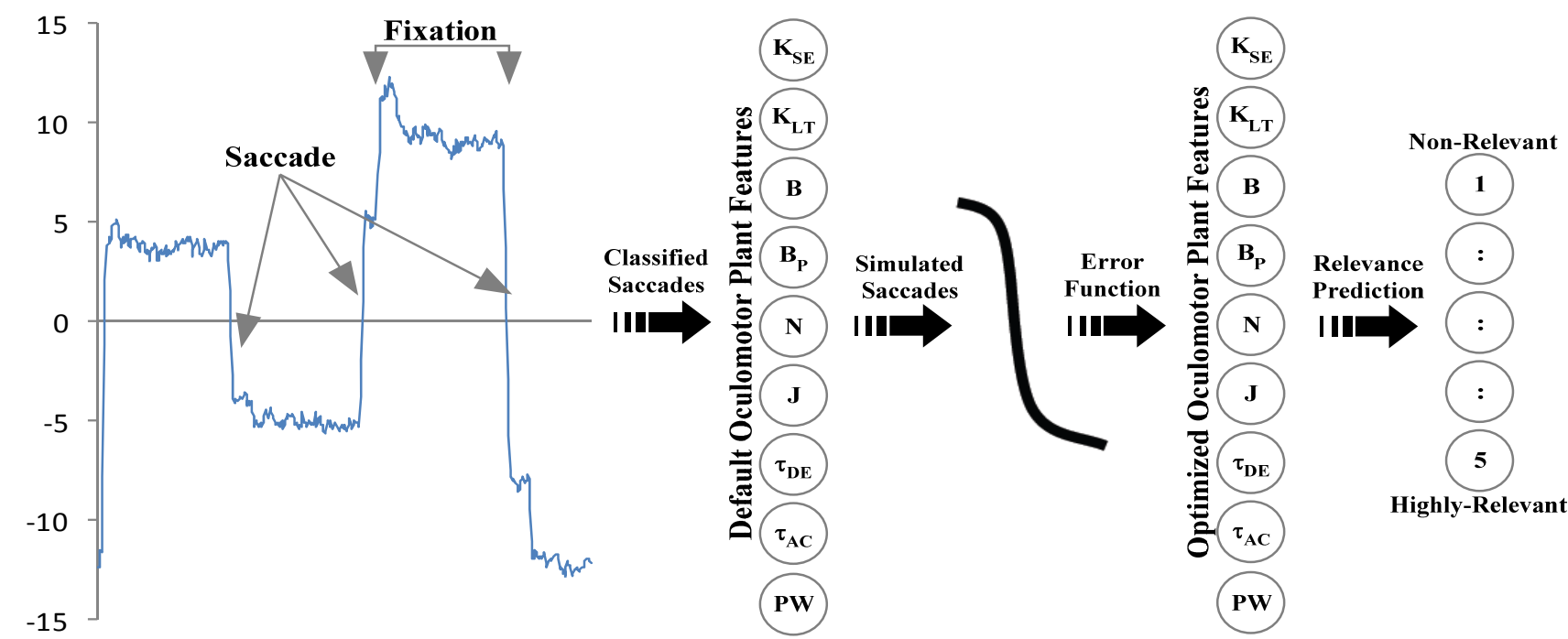

Figure 2. Raw eye movement signal before classification (Left). OPF vector default values and the simulated saccade trajectory (Middle). Optimized OPF vector and Relevance prediction task (Right)

to another) can be analyzed to derive the users attention patterns. The retinal image is transmitted to the brain during fixations but not during saccades. The static and dynamic characteristics of the human OP are represented by the series elasticity, viscosity, active-state tension, length-tension and force-velocity relationships and the frequency characteristics of the neuronal control signal sent by the brain to the extraocular muscles and the speed of propagation of this signal.

We next present details of the underlying oculomotor plant features, the integration of these features into an existing interest modeling architecture and then how the features are expected to provide value during user modeling.

\subsection{Oculomotor Plant Features (OPF)}

The following subset of oculomotor plant features was empirically selected as a feature-set to represent saccadic eye movement during both horizontal and vertical trajectories. The 18-parameter OPF listed below includes, series elasticity resistive properties of an eye muscle, length-tension - the relationship between the length of an extraocular muscle and the force it is capable of exerting, force velocity - the relationship between the velocity of an extraocular muscle during extension/contraction and the force it is capable of exerting, passive viscosity - damping component coefficient for the viscosity of the eye-orbit and surrounding tissues, muscle tension - that ensures an equilibrium state during an eye fixation at primary eye position including the tension slope, neuronal pulse-step - represented as neuronal discharge from the brain.

More information about the 2D Oculomotor Plant and its mathematical derivation of the features can be found in [13].

1. Series Elasticity $(\mathrm{AG})\left[\mathrm{K}_{\mathrm{AG} \_\mathrm{SE}}=2.5 \mathrm{~g} /{ }^{\circ}\right]$

2. Series Elasticity $(\mathrm{ANT})\left[\mathrm{K}_{\mathrm{ANT} \mathrm{SE}}=2.5 \mathrm{~g} /{ }^{\circ}\right]$

3. Length-Tension Relationship (AG) $\left[\mathrm{K}_{\mathrm{AG}_{-} \mathrm{LT}}=1.2 \mathrm{~g} /{ }^{\circ}\right]$

4. Length-Tension Relationship (ANT) $\left[\mathrm{K}_{\mathrm{ANT} \_ \text {LT }}=1.2 \mathrm{~g} /{ }^{\circ}\right]$

5. Force-Velocity Relationship $(\mathrm{AG})\left[\mathrm{B}_{\mathrm{AG}}=0.046 \mathrm{~g} \bullet \mathrm{s} /{ }^{\circ}\right]$

6. Force-Velocity Relationship $(\mathrm{ANT})\left[\mathrm{B}_{\mathrm{ANT}}=0.022 \mathrm{~g} \bullet \mathrm{s} /{ }^{\circ}\right]$

7. Passive Viscosity $\left[\mathrm{B}_{\mathrm{P}}=0.06 \mathrm{~g} \cdot \mathrm{s} /{ }^{\circ}\right]$

8. Tension Slope $(\mathrm{AG})\left[\mathrm{N}_{\mathrm{AG}_{-} \mathrm{C}}=0.8 \mathrm{~g}\right]$

9. Tension Slope (ANT) $\left[\mathrm{N}_{\mathrm{ANT} C \mathrm{C}}^{-}=0.5 \mathrm{~g}\right]$

10. Inertial Mass $\left[\mathrm{J}=0.00004 \overline{\mathrm{g}} \mathrm{g} \cdot \mathrm{s} 2 /^{\circ}\right]$
11. Activation Time $(\mathrm{AG})\left[\tau_{\mathrm{AG} \_\mathrm{AC}}=11.7\right]$

12. Activation Time $(\mathrm{ANT})\left[\tau_{\mathrm{ANT} \_\mathrm{AC}}=2.4\right]$

13. Deactivation Time (AG) $\left[\tau_{\mathrm{AG} D E}=2.0\right]$

14. Deactivation Time $(\mathrm{ANT})\left[\tau_{\mathrm{ANT} \text { DE }}=1.9\right]$

15. Tension Intercept $\left[\mathrm{N}_{\mathrm{FIX} \mathrm{C}_{\mathrm{C}}}=14.0 \mathrm{~g}\right]$

16. Neural Pulse (AG) $\left[\mathrm{N}_{\mathrm{AG} \_\mathrm{SAC}}=55 \mathrm{~g}\right]$

17. Neural Pulse (ANT) $\left[\mathrm{N}_{\text {ANT SAC }}=0.5 \mathrm{~g}\right]$

18. Neural Pulse Width $[\mathrm{PW}=|\mathrm{A}|+6 \mathrm{~ms}]$

Numbers in the brackets represent default values, and $\mathrm{g}-$ grams, $\mathrm{s}$ - seconds, ${ }^{\circ}$ - degrees of the visual angle, $\mathrm{A}$ - amplitude of the recorded saccade. The agonist (AG) muscle contracts, rotates the eye globe and stretches the antagonist (ANT) muscle.

\subsection{Oculomotor Plant Module}

By estimating oculomotor plant features (OPF) via the recorded eye position signal, these OPF values can alter how gaze data is incorporated into the evolving user interest model.

We next present (see Figure 2) the step-by-step process of obtaining OPF vectors and then applying these in the user modeling system to predict relevance values.

During a search task, the recorded eye movement signal from the user is supplied to the Eye Movement Classification module that classifies these position signals into fixations and saccades. We employ Velocity-Threshold (I-VT) algorithm to automatically and reliably identify and classify each recorded saccade's approximate beginning (onset) and ending (offset) from the noisy eye position signal. A threshold of $70 \%$ is empirically selected to split the eye movement recording into fixations and saccades. The detected saccades trajectories represented by the onset and offset coordinates of the eye position and amplitude (length) of the saccade are used in the Oculomotor Plant Mathematical Model (OPMM) [11], which generates simulated saccade trajectories based on the default oculomotor plant feature (OPF) values. Each individual classified saccade is then compared with the simulated saccade and the corresponding OPF values are extracted by minimizing the error between each pair. After several iterations, these optimum OPF feature vectors are then supplied to the user interest estimation module for relevance prediction. 


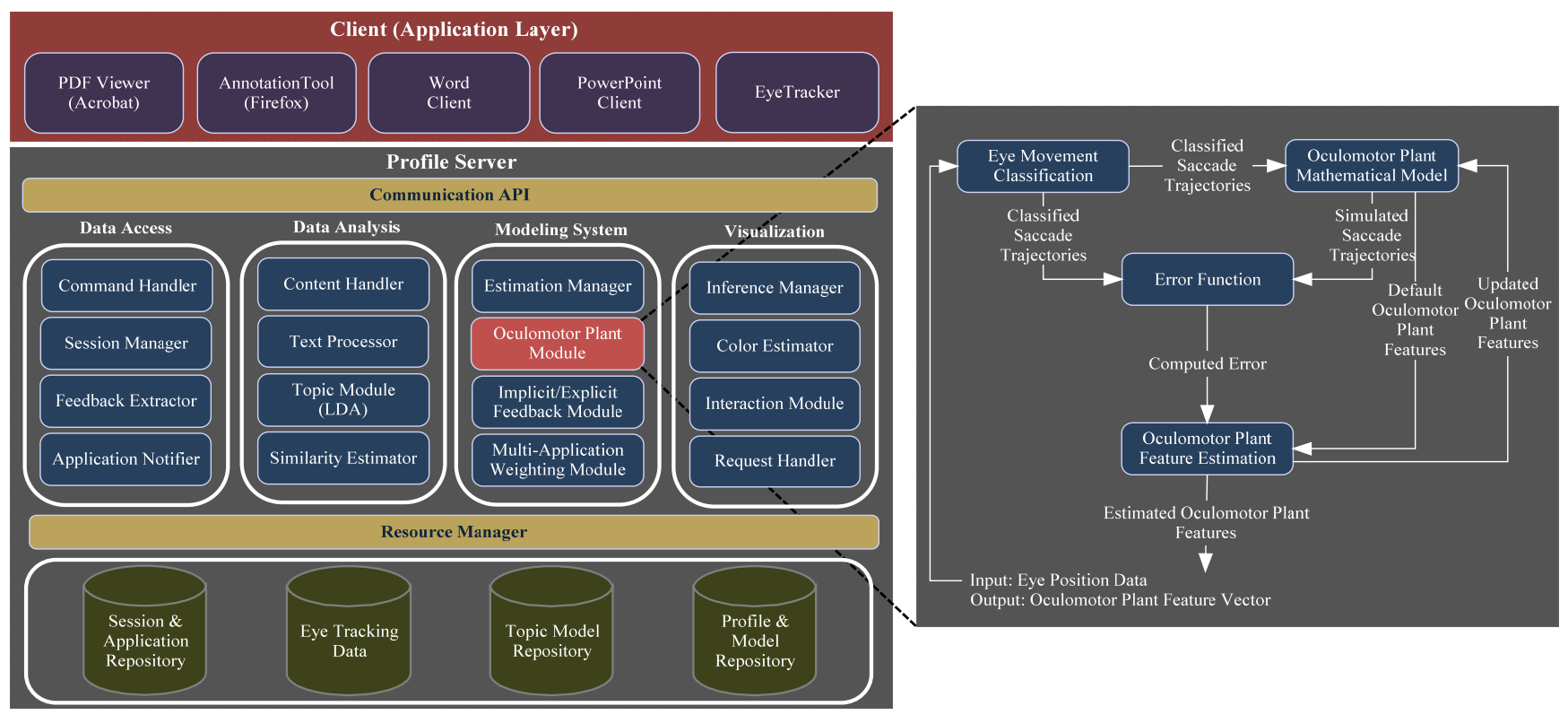

Figure 3. User Modeling Architecture (Left), Oculomotor Plant Module (Right)

\subsection{Can OPF Reveal Relevance?}

Eye movement fixations provide information about attentional states because the only way to acquire information visually is by placing the eye gaze position on the location [6]. It is also possible to measure the pupil dilation to infer the relevance on content with increase pupil dilation indicates fixations on relevant information content. A saccade itself is not an effective source of relevance, since visual perception is suppressed during a saccadic movement where eye is in motion. However, fixations require preceding saccades help to place the gaze in the stimuli to gather information from the target location. We believe that these small but significant movements can provide important cumulative clues in order to understand the underlying deployment of visual attention.

A variety of research in non-human primates has shown that executing saccades quicken when they are employed to a rewarded (positive outcome) location compared to an alternative unrewarded (non-positive outcome) locations [22]. These findings implicate an important role in cognitive learning, cognitive load and reinforcements in order to execute saccades to both rewarding and unrewarding target locations. Studies of task-driven saccades in the absence of a clear visual clue exhibited through slower eye movements [18]. This finding suggests existence of larger cognitive load to identify a cue and then calculate necessary target location to send the gaze position. Compared to an alternative simple eye movement to a visual clue, higher cognitive load may costs oculomotor system and possibly, loss of saccadic speed and accuracy. If these cognitively demanding decisions do influence the movement trajectories of saccades in simple tasks, a natural next step is to look into how relevance may be reflected in oculomotor plant features in more challenging search tasks.

\section{AN OPF-AWARE USER INTEREST MODELING SERVER}

Our interest modeling system [12] incorporates user activity data from multiple applications within a personal profile server (see Figure 3) to support personalized information presentations. The user activity data collected includes a combination of implicit and semi-explicit interest information. It also shares the inferred user interests with registered applications that ask for it.

The profile server currently communicates with a web browser (Mozilla-Firefox) to present search results and also to visualize recommendations, and three other content reading and authoring applications: a PDF reader, Microsoft Word, and Microsoft PowerPoint. Records of user activity in PDF documents, Mozilla Firefox, MS Word and MS PowerPoint are stored in the server and drive the visualizations that the server generates for each of the applications registered for relevant notification request.

The Oculomotor Plant module provides OPF features that are expected to provide insight into the user's assessment of content to the modeling system. The eye tracker provides a continuous eye movement signal during the user activity and (as shown in the Resource Layer of Figure 3) an eye tracking data repository saves session data to be used for relevance prediction. An interest profile is made up of the aggregated heterogeneous interest evidence collected from these different clients that is generated based on all the features, including the new OPF features. The server defines the XML communication interface so that other application clients can interact via TCP/IP. A detailed description of the server and its modules are beyond the scope of this paper and can be found in [12].

\section{FUTURE WORK: GATHERING OPF DATA FOR REAL INFORMATION TASKS} Saccades are integral parts of visual attention. The above framework and architecture incorporates OPF data capture, storage and analysis techniques to explore the value of saccades in determining the relevance of encountered content. Going forward, we will perform controlled studies to capture OPF data during information gathering tasks. By giving the user a specific search task and a set of documents of known relevance, we can initially explore the hypothesis that OPF data will be distinguishable between the relevant and non-relevant documents. The subjects' task will be to (i) read the search task assigned and decide whether each document associated with the task is relevant to the task assignment. (ii) Select the relevance score for each document on a 5-point scale (1-Non-Relevent, 5Higly Relevant), for content relevance assessment. Eye 
movements will be recorded during reading the associated documents. The resulting data will lead to an assessment of if and how OPF data can be used as implicit relevance feedback in future systems.

\section{DISCUSSION AND CONCLUSION}

We have introduced a framework for enhancing implicit relevance feedback by including oculomotor plant features during the use of eye tracking data. In order to further evaluate the proposed architecture, we are currently designing a multiapplication user study that will gather user activity data, including eye tracking data, and post-task relevance judgments from users. This will allow us to assess user behavior in relation to the relevance of documents in order to build and evaluate the possibility of using oculomotor plant features in user interest modeling context. We believe that understanding how the OPF values change over the course of user engagement with information resources will be valuable in the pursuit of developing more accurate user interest models.

There is a wide range of follow-on work related to the user modeling approach explored here. We limited our approach to features that could be computed from oculomotor plant data. Understanding how saccades relate to the fixations they precede is also an important part of moving towards a richer account of relevance learning in the context of user modeling. Most theories and models are based on fixation patterns that lack an understanding of how saccadic eye movements preceding these fixations are affected by the task environment.

\section{ACKNOWLEDGMENTS}

This work was supported in part by National Science Foundation grant DUE-0938074.

\section{REFERENCES}

[1] Borji, A. and Itti, L., "Defending Yarbus: Eye movements reveal observers' task," Journal of vision, vol. 14, p. 29, 2014.

[2] Borji, A., Lennartz, A., and Pomplun, M., "What do eyes reveal about the mind?: Algorithmic inference of search targets from fixations," Neurocomputing, vol. 149, pp. 788-799, 2015.

[3] Borji, A., Sihite, D. N., and Itti, L., "Probabilistic learning of task-specific visual attention," in Computer Vision and Pattern Recognition (CVPR), 2012 IEEE Conference on, 2012, pp. 470-477.

[4] Buscher, G., Dengel, A., and van Elst, L., "Eye movements as implicit relevance feedback," in CHI'08 extended abstracts on Human factors in computing systems, 2008, pp. 2991-2996.

[5] Coddington, J., Xu, J., Sridharan, S., Rege, M., and Bailey, R., "Gaze-based image retrieval system using dual eyetrackers," in Emerging Signal Processing Applications (ESPA), 2012 IEEE International Conference on, 2012, pp. 37-40.

[6] Cole, M. J., Gwizdka, J., Bierig, R., Belkin, N. J., Liu, J., Liu, C., et al., "Linking search tasks with low-level eye movement patterns," in Proceedings of the 28th Annual European Conference on Cognitive Ergonomics, 2010, pp. 109-116.

[7] Goldberg, J. and Helfman, J., "Eye tracking for visualization evaluation: Reading values on linear versus radial graphs," Information visualization, 2011.
[8] Haji-Abolhassani, A. and Clark, J. J., "A computational model for task inference in visual search," Journal of vision, vol. 13, p. 29, 2013.

[9] Hussain, Z., Klami, A., Kujala, J., Leung, A. P., Pasupa, K., Auer, P., et al., "PinView: Implicit Feedback in Content-Based Image Retrieval," arXiv preprint arXiv:1410.0471, 2014.

[10] Iqbal, S. T. and Bailey, B. P., "Using eye gaze patterns to identify user tasks," in The Grace Hopper Celebration of Women in Computing, 2004, pp. 5-10.

[11] Jayarathna, S., "Two Dimentional Oculomotor Plant Mechanical Model (2DOPMM)," Texas State UniversitySan Marcos, 2010.

[12] Jayarathna, S., Patra, A., and Shipman, F., "Unified Relevance Feedback for Multi-Application User Interest Modeling," presented at the Proceedings of the 15th ACM/IEEE-CS Joint Conference on Digital Libraries, Knoxville, Tennessee, USA, 2015.

[13] Komogortsev, O. V., Holland, C., and Jayarathna, S., "Two-Dimensional Linear Homeomorphic Oculomotor Plant Mathematical Model," 2012.

[14] Komogortsev, O. V., Jayarathna, S., Aragon, C. R., and Mahmoud, M., "Biometric identification via an oculomotor plant mathematical model," in Proceedings of the 2010 Symposium on Eye-Tracking Research \& Applications, 2010, pp. 57-60.

[15] Komogortsev, O. V., Karpov, A., Price, L. R., and Aragon, C., "Biometric authentication via oculomotor plant characteristics," in Biometrics (ICB), 2012 5th IAPR International Conference on, 2012, pp. 413-420.

[16] Kozma, L., Klami, A., and Kaski, S., "GaZIR: gaze-based zooming interface for image retrieval," in Proceedings of the 2009 international conference on Multimodal interfaces, 2009, pp. 305-312.

[17] Leigh, R. J. and Zee, D. S., The neurology of eye movements: Oxford University Press, 2015.

[18] McColeman, C. M. and Blair, M. R., "Task relevance moderates saccade velocities to spatially separated cues."

[19] Plumlee, M. D. and Ware, C., "Zooming versus multiple window interfaces: Cognitive costs of visual comparisons," ACM Transactions on Computer-Human Interaction (TOCHI), vol. 13, pp. 179-209, 2006.

[20] Rajashekar, U., Bovik, A. C., and Cormack, L. K., "Visual search in noise: Revealing the influence of structural cues by gaze-contingent classification image analysis," Journal of Vision, vol. 6, p. 7, 2006.

[21] Rayner, K., "Eye movements and cognitive processes in reading, visual search, and scene perception," Studies in Visual Information Processing, vol. 6, pp. 3-22, 1995.

[22] Takikawa, Y., Kawagoe, R., Itoh, H., Nakahara, H., and Hikosaka, O., "Modulation of saccadic eye movements by predicted reward outcome," Experimental brain research, vol. 142, pp. 284-291, 2002.

[23] Yarbus, A. L., Haigh, B., and Rigss, L. A., Eye movements and vision vol. 2: Plenum press New York, 1967.

[24] Zelinsky, G. J., Peng, Y., and Samaras, D., "Eye can read your mind: Decoding gaze fixations to reveal categorical search targets," Journal of vision, vol. 13, p. 10, 2013. 